\title{
Biocompatible, Free-Standing Film Composed of Bacterial Cellulose Nanofibers/Graphene Composite
}

Lin Jin, ${ }^{\dagger, t}$ Zhiping Zeng, ${ }^{\S}$ Shreyas Kuddannaya, ${ }^{\S}$ Dingcai Wu,${ }^{\ddagger *}$ Yilei Zhang, ${ }^{\S} *$

Zhenling Wang $^{\dagger *}$

${ }^{\dagger}$ The Key Laboratory of Rare Earth Functional Materials and Applications, Zhoukou

Normal University, Zhoukou 466001, P. R. China

*Materials Science Institute, PCFM Lab and DSAPM Lab, School of Chemistry and

Chemical Engineering, Sun Yat-sen University, Guangzhou 510275, P. R. China.

${ }^{\S}$ School of Mechanical \& Aerospace Engineering, Nanyang Technological

University, 50 Nanyang Avenue, Singapore 639798, Singapore

\section{AUTHOR INFORMATION}

Dr. L. Jin, Prof. Z. L. Wang*

${ }^{\dagger}$ The Key Laboratory of Rare Earth Functional Materials and Applications, Zhoukou

Normal University, Zhoukou 466001, P. R. China

E-mail:zlwang2007@,hotmail.com;

Fax: 86394-8178518; Tel: 86394-8178996

Prof. D. C. $\mathrm{Wu}^{*}$

${ }^{\ddagger}$ Materials Science Institute, School of Chemical and Chemical Engineering, Sun

Yat-sen University, Guangzhou, 510275, P. R. China

E-mail:wudc@mail.sysu.edu.cn.

Z. P. Zeng, S. Kuddannaya, Prof. Y. L. Zhang* 
${ }^{\S}$ School of Mechanical \& Aerospace Engineering, Nanyang Technological University,

50 Nanyang Avenue, Singapore 639798, Singapore

E-mail:ylzhang@ntu.edu.sg 
Figure S1. (A) Typical AFM image and (B) the corresponding height profile of the as-prepared GO sheets.
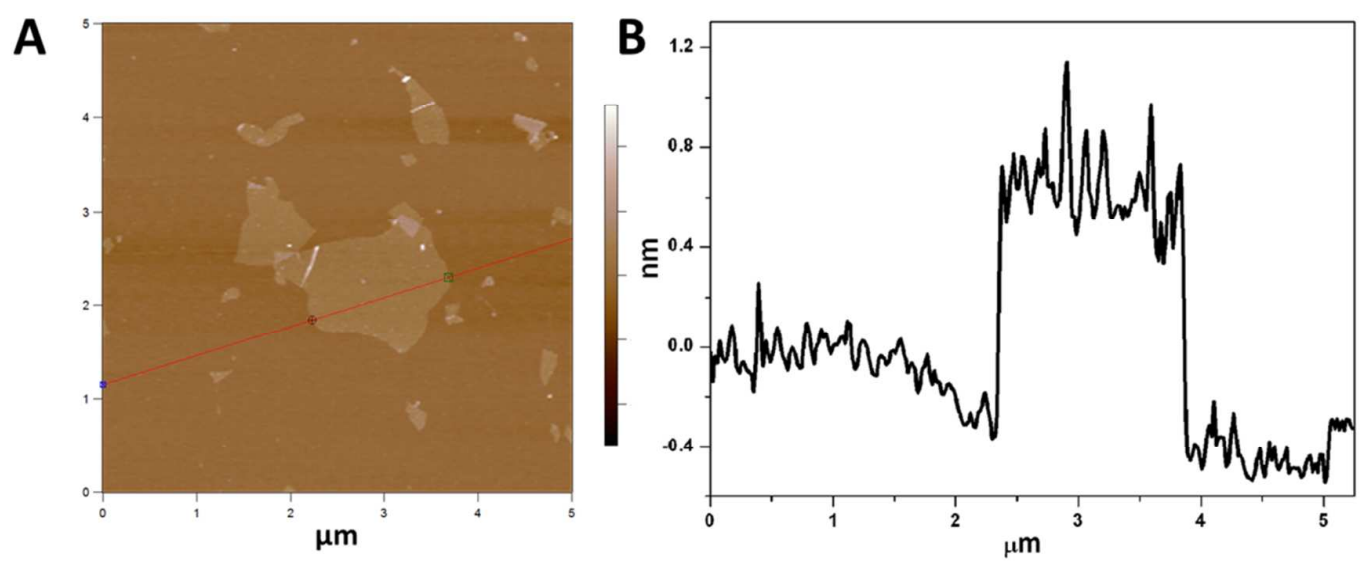
Figure S2. The SEM images of BC-RGO film.
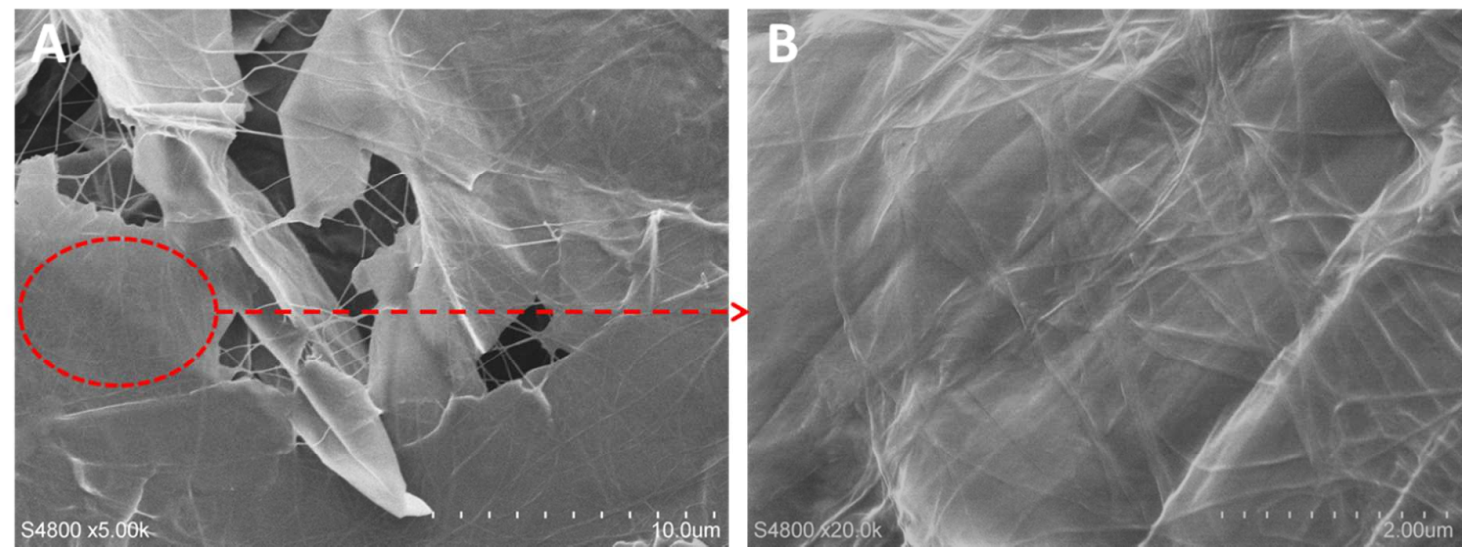
Figure S3. The SEM image of BC-RGO film with positive side.

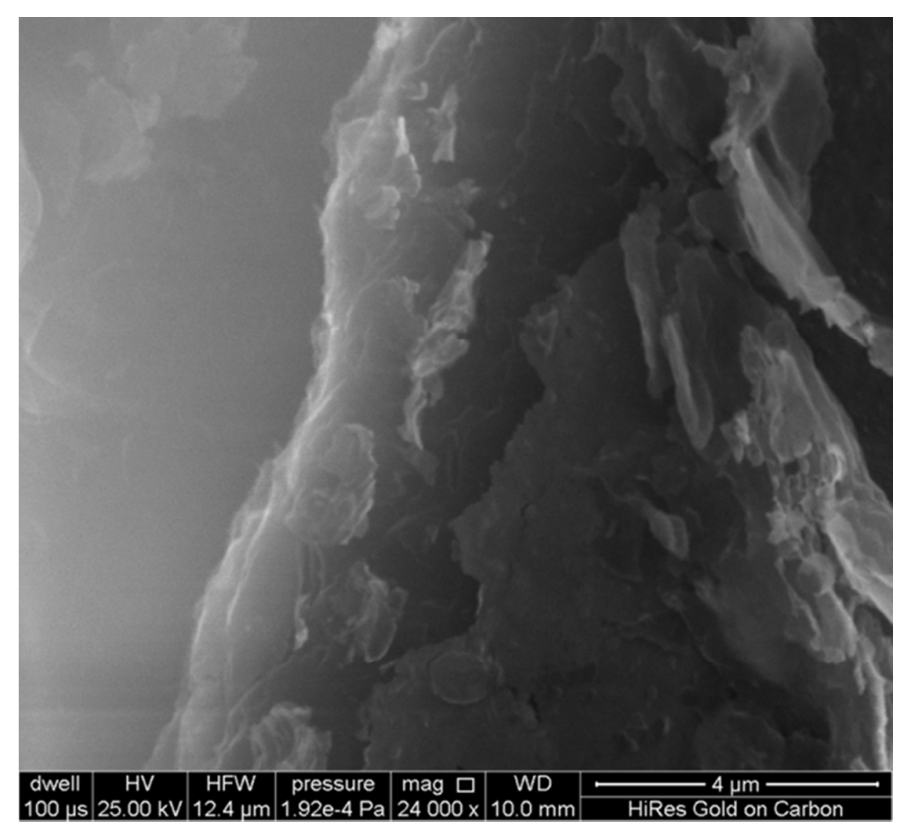

